\title{
Tamiflu still prudent for pandemic influenza, PHAC maintains
}

Published at www.cmaj.ca on Dec. 23, 2009

$\mathrm{T}$ he Public Health Agency of Canada (PHAC) is standing by its decision to stockpile and widely distribute neuraminidase inhibitors such as oral oseltamivir (Tamiflu) for use against the pandemic (H1N1) 2009 influenza, despite an updated Cochrane review that questions their usefulness.

The review assessed 20 trials to see how well neuraminidase inhibitors prevented or reduced the symptoms of influenza, its transmission and complications in healthy adults (BMJ 2009;339:b5106). It also assessed the frequency of adverse events and concluded that while the drugs reduced the symptoms of influenza by about a day, they do not prevent infection or stop viral spread. The authors questioned the reliability of evidence that the drugs reduce the risk of complications in healthy adults and advised against prescribing them to treat seasonal influenza.

In an accompanying editorial, the British Medical Journal argued that as public health agencies had stockpiled oseltamivir on the basis of the manufacturer's claims that it reduced the risk of complication in healthy adults, it is imperative that vaccine manufacturer Roche make available the data from eight unpublished trials.

But PHAC indicated that the controversy is immaterial to its recommendations. "The articles published in the $B M J$ do not change [the] Public Health Agency of Canada's advice with respect to the use of antivirals with the H1N1 flu virus," PHAC said in a written response to $C M A J$ inquiries. PHAC also noted that the World Health Organization has indicated it would not vary its advice about using antivirals.

The Cochrane review focused on seasonal influenza, not the pandemic (H1N1) 2009 virus, the agency pointed out. It said antivirals are not recom-

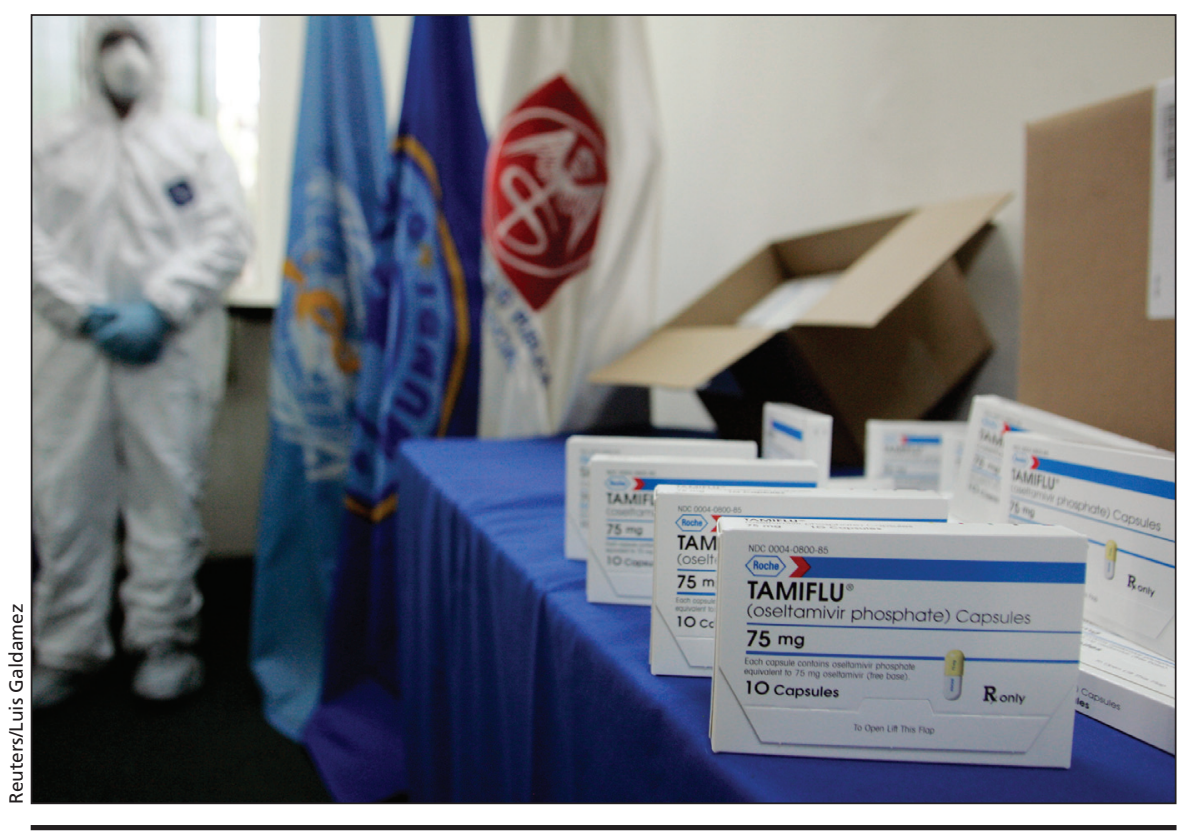

A Cochrane review has concluded that while oral oseltamir (Tamiflu) reduces the symptoms of influenza by a day, it does not prevent infection or stop viral spread.

mended for treating healthy adults with mild illness, but continued to recommend them for those at risk of complications, including young children, pregnant women, people with chronic medical conditions and patients who are "very ill."

Leading Canadian infectious disease experts backed PHAC's decision to stockpile the drug and make it widely available. They also concurred on the reviewers' call for more studies.

Given that the drug is safe, cheap and has some impact on the severity and duration of symptoms, Dr. Allison McGeer believes it makes sense to use it.

"Faced with the same data and the same decision again, I think people should be stockpiling," adds McGeer, director of infection control at the Mount Sinai Hospital in Toronto, Ontario.

It's difficult to get good data about reductions in complications in an outpatient population, which is where most people are taking oseltamivir, says McGeer, who also questioned the
Cochrane review's interpretation of the data about complications.

In the review, principal investigator Dr. Tom Jefferson and colleagues noted a reduction in complications that is not statistically significant in a metaanalysis, but is clinically significant, says McGeer. The data implies that if patients are treated within 48 hours of the onset of symptoms, they are 55\% less likely to have complications than if they were not treated.

"When you've done studies that demonstrate a $55 \%$ reduction in complications, the message is not that they (the drugs) don't work — the message is that we want more data," she says.

The main problem for doctors treating influenza patients with the antivirals is that they are very effective if patients come for treatment in the first $6-12$ hours, and generally effective for up to 48 hours. But many people don't come in for treatment that early, McGeer says.

McGeer and Dr. Fred Aoki, an infectious disease physician at the Winnipeg 
Health Sciences Centre and an influenza researcher at the University of Manitoba in Winnipeg argued that the negative interpretation of the data was a function of under-powered studies, which took place during years where there was no severe influenza strain.

Aoki believes that once the data is analyzed from the most recent outbreak, it will show that treating with oseltamivir does reduce complications. "It's my expectation that oseltamivir should work against this pandemic virus."

The trick will be to get it into the hands of people more quickly than during the first 36-48 hours of their illness, he adds.

Stockpiling it was the right thing to do, Aoki says. "We have to take out this insurance policy against there being unpredictable catastrophic disease." But he adds that emerging information about resistance to oseltamivir means that PHAC should consider replacing the stockpile of oseltamivir with zanamivir, an inhaled antiviral. Laura Eggertson, Ottawa, Ont.

DOI:10.1503/cmaj.109-3145 